Supporting Information

\title{
Bioinspired Ultrafast-Responsive Nanofluidic System for Ion and Molecule Transport with Speed Control
}

Can Wang, ${ }^{\dagger, \ddagger}$ Dianyu Wang, ${ }^{\S}$ Weining Miao, ${ }^{\dagger, \ddagger}$ Lianxin Shi, ${ }^{\dagger, \ddagger}$ Shutao Wang, ${ }^{\dagger, \ddagger}$ Ye Tian, ${ }^{*},+\ddagger$ and Lei Jiang ${ }^{\dagger, \ddagger}$

${ }^{\dagger}$ Key Laboratory of Bioinspired Smart Interfacial Science, Technical Institute of Physics and Chemistry, Chinese Academy of Sciences, Beijing 100190, People’s Republic of China

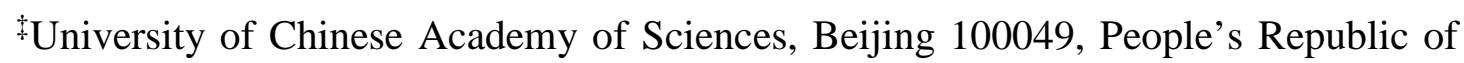
China

${ }^{\S}$ Beihang University, Beijing 100191, People’s Republic of China

*E-mail: tianyely@iccas.ac.cn 

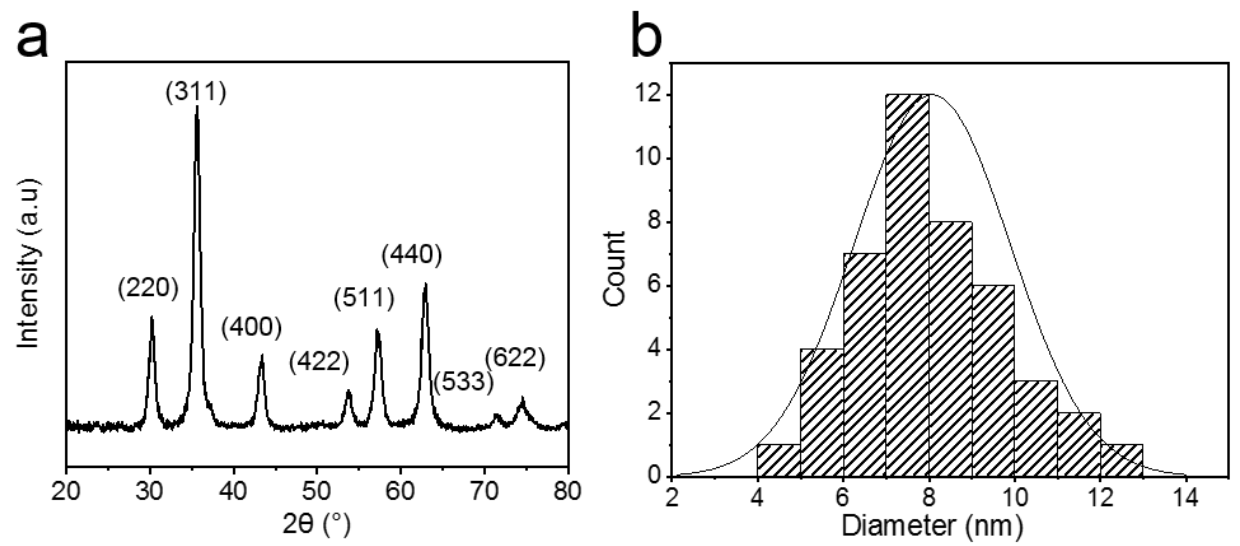

Figure S1. The structure characterization of the used ferrofluids. (a) The XRD pattern of the ferrofluids. (b) The particle size distribution of the ferrofluids from the TEM image.
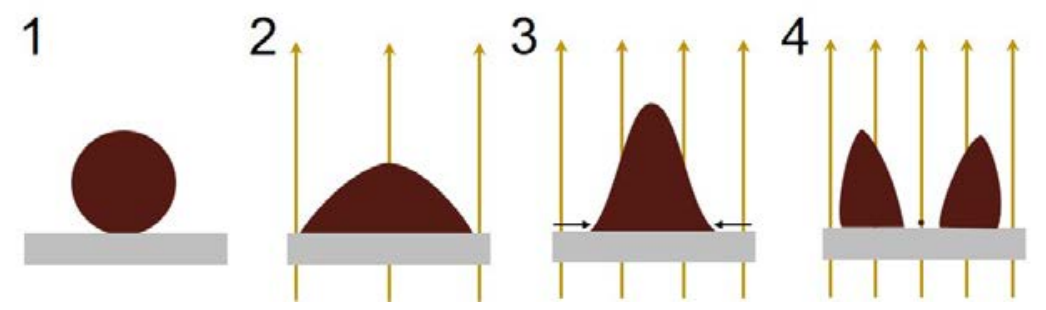

\section{5}

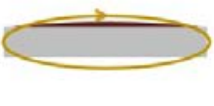

Figure S2. Schematic side view of a droplet ferrofluids in the different magnetic fields (dark yellow lines, magnetic field; brown areas, ferrofluids; grey areas, AAO membranes). State of the droplet is indicated as follows: 1, without magnetic field (nearly spherical droplet); 2, weak field (slightly deformed droplet); 3, strong field (conical spiked droplet); 4, above critical field (two daughter droplets); 5, apply lateral magnetic field (flat ferrofluids film). 

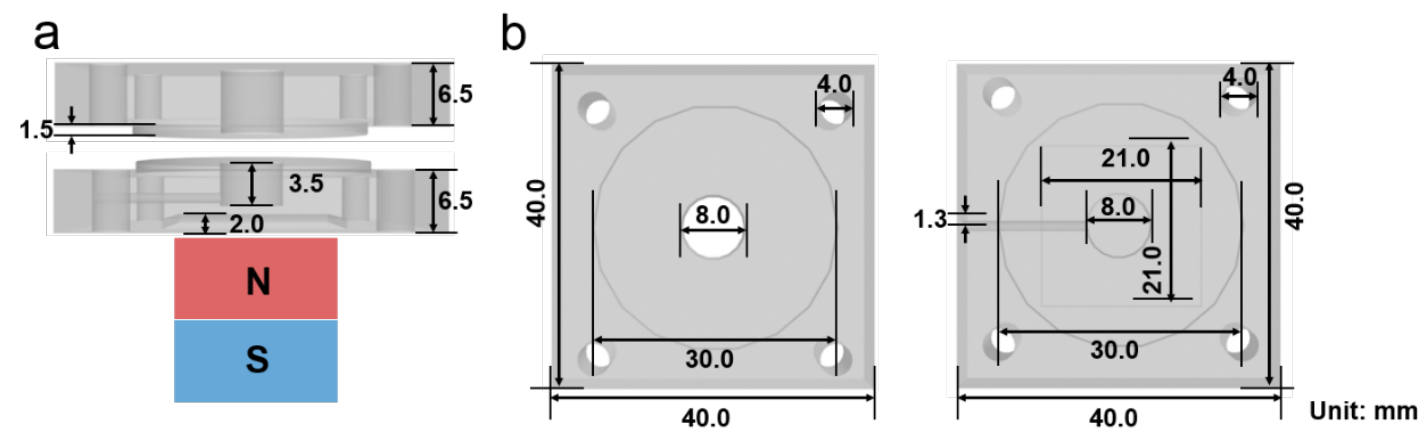

Figure S3. Design of the homemade plexiglass molds for the magnetically tunable gating system. (a) Side view. (b) Forward view. Unit: mm.

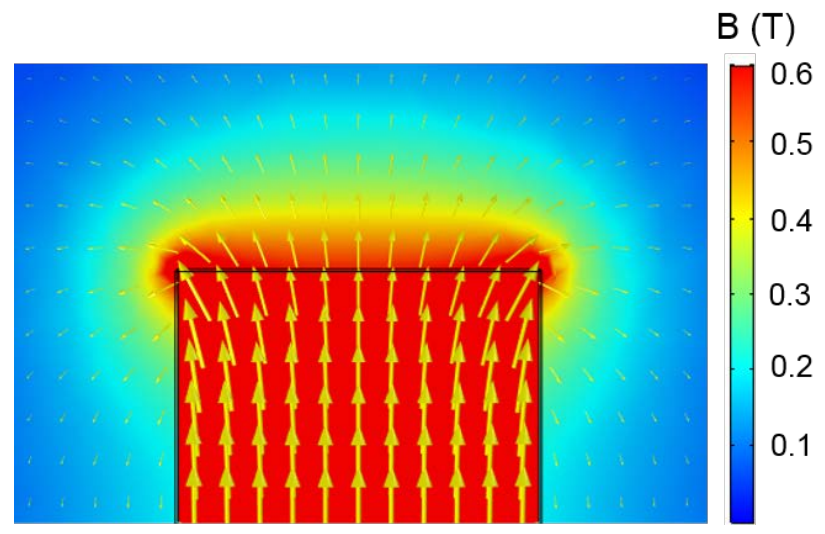

Figure S4. Schematic illustration of the magnetic field intensity distribution on the surface of a rectangular permanent magnet.

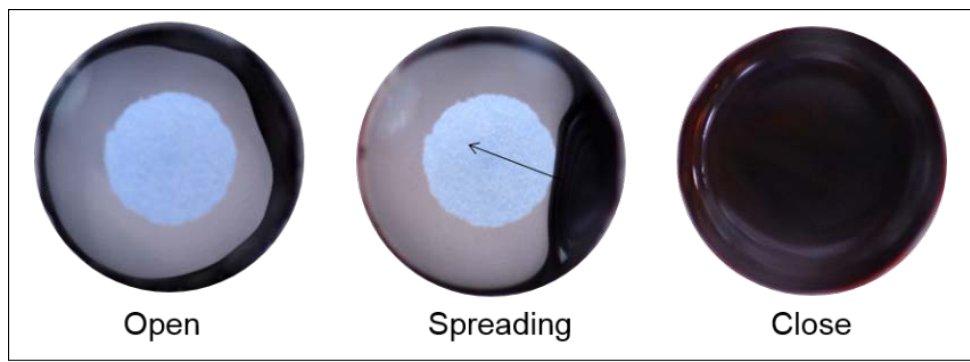

Figure S5. When the superhydrophilic nanofluidic system was switched from the open state to the closed state, three optical photographs of the ferrofluids during this process. 
a

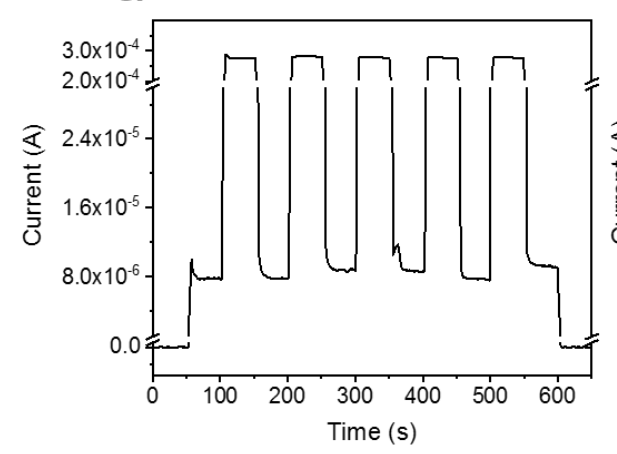

b

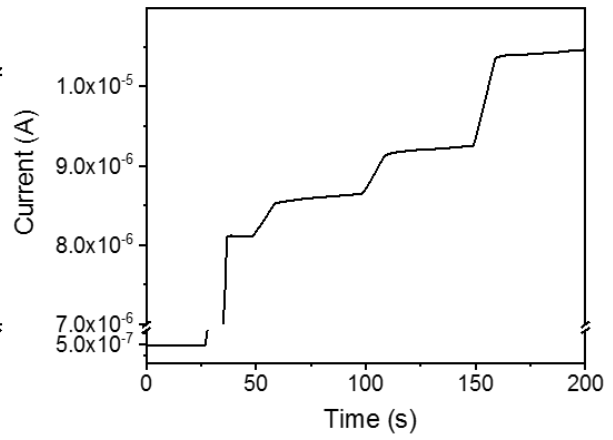

Figure S6. Multilevel gating of superhydrophilic nanofluidic system. (a) In-situ reversible switching between closed, partly open, and open states was achieved by regulating the external magnetic field, and the duration of each state was the $50 \mathrm{~s}$. (b) Changing the external magnetic field in-situ makes the partly open state exhibit transmembrane currents of different orders of magnitude.

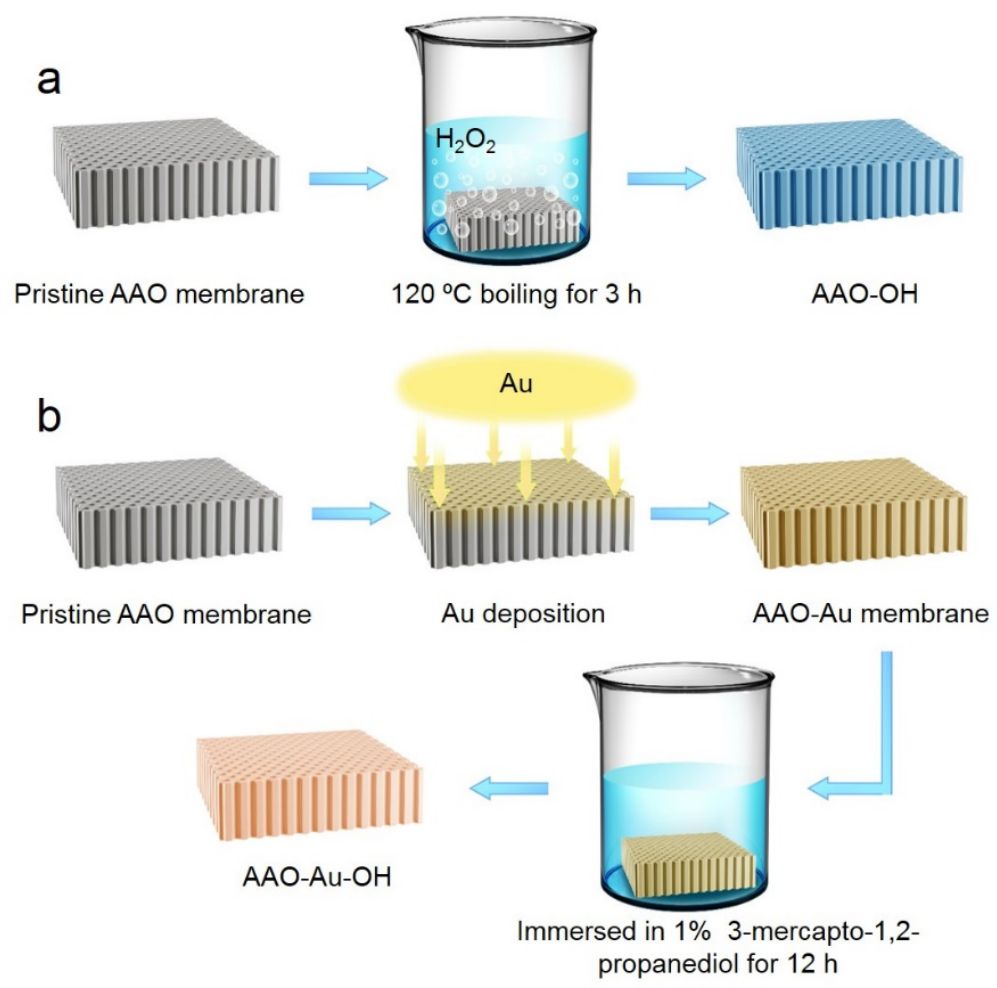

Figure S7. Modification processes of the superhydrophilic AAO membrane. (a) Immerse the pristine AAO membrane in the $\mathrm{H}_{2} \mathrm{O}_{2}$ solution at $120^{\circ} \mathrm{C}$ for $3 \mathrm{~h}$ to obtain 
superhydrophilic AAO membranes. (b) An AAO-Au membrane was obtained by depositing a layer of gold on the AAO membrane using electron beam evaporation technology. Then the membrane was immersed in a 1\% 3-mercapto-1,2-propanediol solution for $12 \mathrm{~h}$, and finally, a superhydrophilic AAO membrane was obtained.

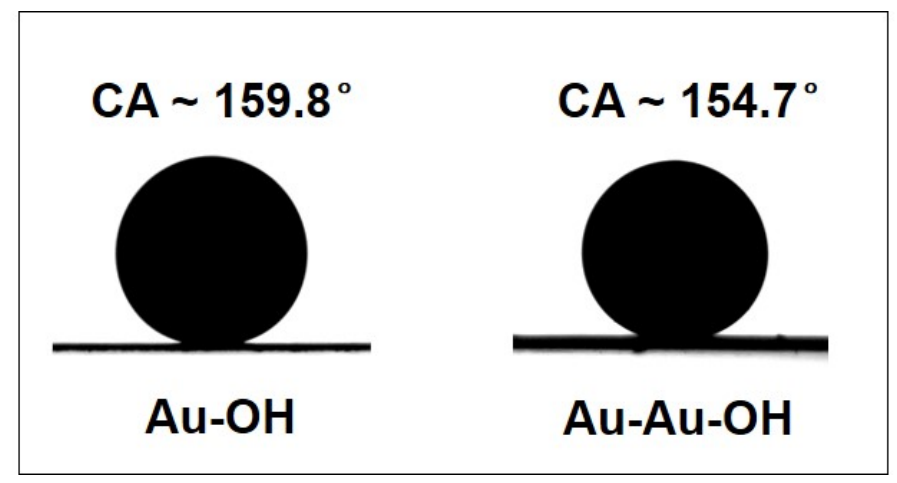

Figure S8. Underwater ferrofluids contact angles of different superhydrophilic AAO membranes. (a) AAO membrane modified by $\mathrm{H}_{2} \mathrm{O}_{2}$. (b) AAO membrane modified by 3-mercapto-1,2-propanediol. 


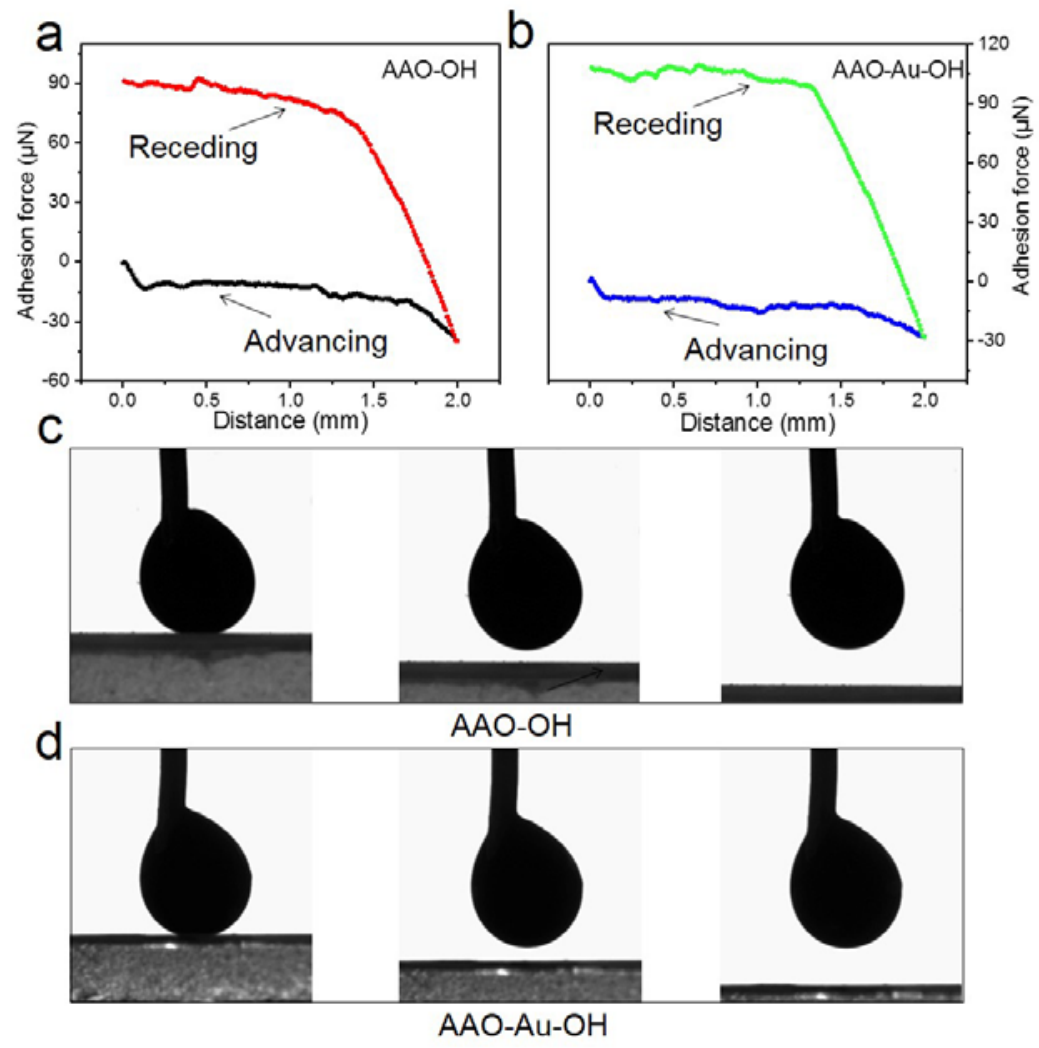

Figure S9. Adhesion measurements. The ferrofluids adhesion on the (a) superhydrophilic AAO membrane modified by $\mathrm{H}_{2} \mathrm{O}_{2}$ and (b) superhydrophilic AAO membrane modified by 3-mercapto-1,2-propanediol. And the (c), (d) corresponding snapshots of the receding process which shows the ferrofluids are completely non-stick on superhydrophilic AAO membranes.
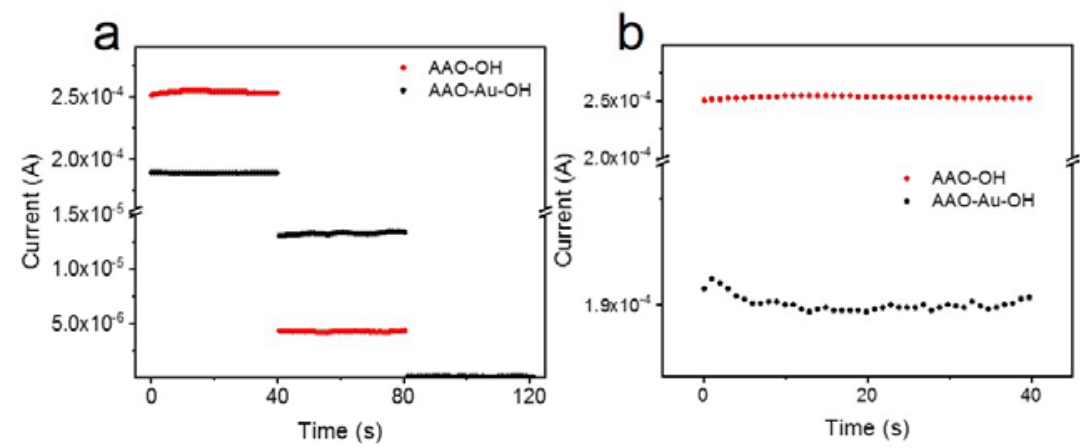

Figure S10. (a) $I-T$ curves of superhydrophilic AAO membranes obtained by two 
different modification methods recorded in the different external magnetic fields. (b)

Enlarged data of transmembrane current at $0-40 \mathrm{~s}$ in (a).

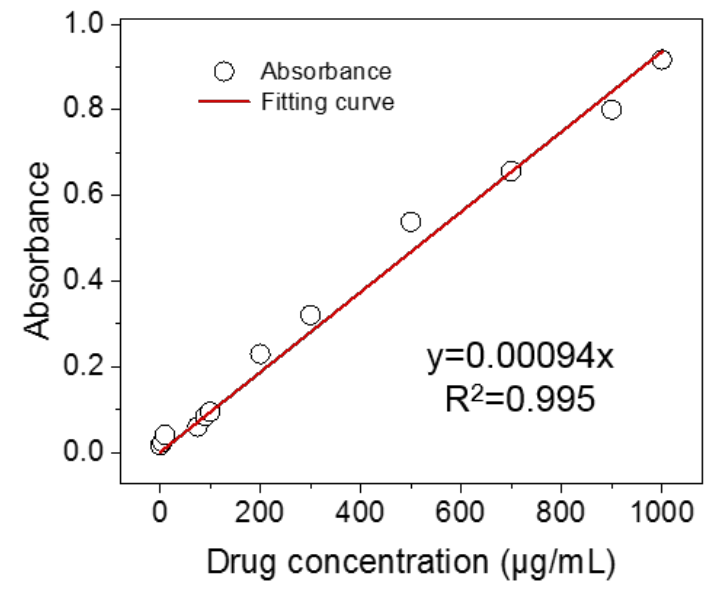

Figure S11. The calibration curve of constructed using the absorbance versus corresponding drug concentrations. 